\title{
An octonionic formulation of the M-theory algebra
}

\section{A. Anastasiou, L. Borsten, M.J. Duff, L.J. Hughes and S. Nagy}

Theoretical Physics, Blackett Laboratory, Imperial College London, London SW' 2AZ, United Kingdom

E-mail: alexandros.anastasiou07@imperial.ac.uk, leron.borsten@imperial.ac.uk, m.duff@imperial.ac.uk, leo.hughes07@imperial.ac.uk, s.nagy11@imperial.ac.uk

ABSTRACT: We give an octonionic formulation of the $\mathcal{N}=1$ supersymmetry algebra in $D=11$, including all brane charges. We write this in terms of a novel outer product, which takes a pair of elements of the division algebra $\mathbb{A}$ and returns a real linear operator on $\mathbb{A}$. More generally, with this product comes the power to rewrite any linear operation on $\mathbb{R}^{n}$ $(n=1,2,4,8)$ in terms of multiplication in the $n$-dimensional division algebra $\mathbb{A}$. Finally, we consider the reinterpretation of the $D=11$ supersymmetry algebra as an octonionic algebra in $D=4$ and the truncation to division subalgebras.

KEYWORDS: Extended Supersymmetry, M-Theory

ArXiv EPRINT: 1402.4649 


\section{Contents}

1 Introduction 1

2 The division algebras 2

3 A new outer product $\quad 3$

4 Octonionic spinors in $D=11 \quad 4$

5 The octonionic M-algebra 5

6 Relation to lower dimensions $\quad 6$

\section{Introduction}

A recurring theme in the study of supersymmetry and string theory is the connection to the four division algebras: the real numbers $\mathbb{R}$, the complex numbers $\mathbb{C}$, the quaternions $\mathbb{H}$ and the octonions $\mathbb{O}$. See, for example, [1-9]. The octonions are of particular interest in this context since they may be used to describe representations of the Lorentz group in spacetime dimensions $D=10,11$, where string and M-theory live. Furthermore, the octonions provide a natural explanation $[10,11]$ for the appearance of exceptional groups as the U-dualities of supergravities [12] and M-theory [13, 14].

A $D=11$ spinor with 32 components may be packaged as a 4-component octonionic column vector $[6,15]$. This has prompted the question of how to write the algebra of $D=11$ supergravity (or 'M-algebra') using octonionic supercharges $Q$. This was explored in [15] where the problem was highlighted that the apparently natural choice of octonionic matrices could not provide enough degrees of freedom to account for all of M-theory's brane charges. Another fundamental question that arises when writing the $\{Q, Q\}$ algebra in this way is whether or not the usual anti-commutator is really the appropriate object to study, given that the fermionic supercharges are written over a non-commutative and non-associative algebra $\mathbb{O}$.

In the present paper we tackle this problem by introducing a novel outer product, which takes a pair of elements belonging to a division algebra $\mathbb{A}$ and returns a real linear operator on $\mathbb{A}$, expressed using multiplication in $\mathbb{A}$. This product enables one to rewrite any expression involving $n \times n$ matrices and $n$-dimensional vectors in terms of multiplication in the $n$-dimensional division algebra $\mathbb{A}$. We solve the problem of the octonionic M-algebra using this product, which allows a derivation of the correct $\{Q, Q\}$ bracket. In the final section we consider "Cayley-Dickson halving" the octonionic M-algebra, which corresponds to its reinterpretation as the maximal supergravity algebra in $D=7,5,4$. For example, the M-algebra may be considered to be an octonionic rewriting of the $D=4, \mathcal{N}=8$ 
supersymmetry algebra; from this perspective the $D=4, \mathcal{N}=1$ algebra comes from a truncation $\mathbb{O} \rightarrow \mathbb{R}$.

\section{The division algebras}

A normed division algebra is an algebra $\mathbb{A}$ equipped with a positive-definite norm satisfying the condition

$$
\|x y\|=\|x\|\|y\| .
$$

Remarkably, there are only four such algebras: $\mathbb{R}, \mathbb{C}, \mathbb{H}$ and $\mathbb{O}$, with dimensions $n=1,2,4$ and 8 , respectively.

A division algebra element $x \in \mathbb{A}$ is written as the linear combination of $n$ basis elements with real coefficients: $x=x_{a} e_{a}$, with $x_{a} \in \mathbb{R}$ and $a=0, \cdots,(n-1)$. One basis element $e_{0}=1$ is real; the other $(n-1)$ basis elements $e_{i}$ are imaginary:

$$
e_{0}^{2}=1, \quad e_{i}^{2}=-1,
$$

where $i=1, \cdots,(n-1)$. In analogy with the complex case, we define a conjugation operation indicated by ${ }^{*}$, which changes the sign of the imaginary basis elements:

$$
e_{0}^{*}=e_{0}, \quad e_{i}^{*}=-e_{i} .
$$

The multiplication rule for the basis elements of a division algebra is given by:

$$
\begin{aligned}
& e_{a} e_{b}=\left(\delta_{a 0} \delta_{b c}+\delta_{0 b} \delta_{a c}-\delta_{a b} \delta_{0 c}+C_{a b c}\right) e_{c} \equiv \Gamma_{b c}^{a} e_{c} \\
& e_{a}^{*} e_{b}=\left(\delta_{a 0} \delta_{b c}-\delta_{0 b} \delta_{a c}+\delta_{a b} \delta_{0 c}-C_{a b c}\right) e_{c} \equiv \bar{\Gamma}_{b c}^{a} e_{c},
\end{aligned}
$$

where we define the structure constants ${ }^{1}$

$$
\begin{aligned}
& \Gamma_{b c}^{a}=\delta_{a 0} \delta_{b c}+\delta_{b 0} \delta_{a c}-\delta_{a b} \delta_{c 0}+C_{a b c}, \\
& \bar{\Gamma}_{b c}^{a}=\delta_{a 0} \delta_{b c}-\delta_{b 0} \delta_{a c}+\delta_{a b} \delta_{c 0}-C_{a b c} \Rightarrow \Gamma_{b c}^{a}=\bar{\Gamma}_{c b}^{a} .
\end{aligned}
$$

The tensor $C_{a b c}$ is totally antisymmetric with $C_{0 a b}=0$, so it is identically zero for $\mathbb{A}=\mathbb{R}, \mathbb{C}$. For the quaternions $C_{i j k}$ is simply the permutation symbol $\varepsilon_{i j k}$, while for the octonions the non-zero $C_{i j k}$ are specified by the set of oriented lines of the Fano plane, see [16].

One of the most important properties of the division algebras is that they provide a representation of the $\mathrm{SO}(n)$ Clifford algebra. This is reflected in the structure constants, which satisfy

$$
\begin{aligned}
\Gamma^{a} \bar{\Gamma}^{b}+\Gamma^{b} \bar{\Gamma}^{a}=2 \delta^{a b} \mathbb{1}, \\
\bar{\Gamma}^{a} \Gamma^{b}+\bar{\Gamma}^{b} \Gamma^{a}=2 \delta^{a b} \mathbb{1} .
\end{aligned}
$$

In other words, we have the interpretation that multiplying a divison algebra element $\psi$ by the basis element $e_{a}$ has the effect of multiplying $\psi$ 's components by the gamma matrix $\bar{\Gamma}^{a}$ :

$$
e_{a} \psi=e_{a} e_{b} \psi_{b}=\Gamma_{b c}^{a} e_{c} \psi_{b}=e_{c} \bar{\Gamma}_{c b}^{a} \psi_{b} .
$$

\footnotetext{
${ }^{1}$ The unusual choice of index structure is for later convenience - see equations (2.6) and (2.7).
} 
This property is essential for many of the applications of division algebras to physics, including that of this paper.

A natural inner product [16] on $\mathbb{A}$ is given by:

$$
\langle x \mid y\rangle=\frac{1}{2}\left(x^{*} y+y^{*} x\right)=x_{a} y_{a} \quad \text { i.e. } \quad\left\langle e_{a} \mid e_{b}\right\rangle=\delta_{a b} .
$$

This is just the canonical inner product on $\mathbb{R}^{n}$.

\section{A new outer product}

It is interesting to see what other linear operations on $\mathbb{R}^{n}$ look like when written in terms of the division-algebraic multiplication rule. This was explored in [17], but we take a different approach here. Consider the following general problem. Given some linear operator on $\mathbb{R}^{n}$ expressed as an $n \times n$ matrix $M_{a b}$, we would like to find an operator $\hat{M}$ on the division algebra $\mathbb{A}$ such that $\hat{M}$ has the effect of multiplying the components of $x=x_{a} e_{a} \in \mathbb{A}$ by $M_{a b}$ :

$$
\hat{M} x \equiv e_{a} M_{a b} x_{b} .
$$

An explicit form for this operator can be found using the inner product above. First we rewrite

$$
\begin{aligned}
M_{a b} & =M_{c d}\left\langle e_{a} \mid e_{c}\right\rangle\left\langle e_{b} \mid e_{d}\right\rangle \\
& =\frac{1}{2} M_{c d}\left\langle e_{a} \mid e_{c}\left(e_{d}^{*} e_{b}\right)+e_{c}\left(e_{b}^{*} e_{d}\right)\right\rangle .
\end{aligned}
$$

Now it is clear that the operator

$$
\hat{M} \equiv \frac{1}{2} M_{c d}\left(e_{c}\left(e_{d}^{*} \cdot\right)+e_{c}\left((\cdot)^{*} e_{d}\right)\right),
$$

where a dot represents a slot for an octonion, has matrix elements

$$
\left\langle e_{a} \mid \hat{M} e_{b}\right\rangle=M_{a b} .
$$

This suggests that we write the outer product for division algebra elements using their multiplication rule, defining:

$$
\begin{aligned}
\times: \mathbb{A} \otimes \mathbb{A} & \rightarrow \operatorname{End}(\mathbb{A}) \\
e_{a} \otimes e_{b} & \mapsto e_{a} \times e_{b} \equiv \frac{1}{2}\left(e_{a}\left(e_{b}^{*} \cdot\right)+e_{a}\left((\cdot)^{*} e_{b}\right)\right) .
\end{aligned}
$$

With the new product comes the power to rewrite any expression involving $n \times n$ matrices and $n$-dimensional vectors in terms of multiplication in the $n$-dimensional division algebra $\mathbb{A}$.

It is useful to note various equivalent ways of writing the outer product above:

$$
\begin{aligned}
e_{a} \times e_{b} & =\frac{1}{2}\left(e_{a}\left(e_{b}^{*} \cdot\right)+e_{a}\left((\cdot)^{*} e_{b}\right)\right) \\
& =\frac{1}{2}\left(\left(\cdot e_{b}^{*}\right) e_{a}+\left(e_{b}(\cdot)^{*}\right) e_{a}\right) \\
& =\frac{1}{2}\left(e_{a}\left(e_{b}(\cdot)^{*}\right)+e_{a}\left(\cdot e_{b}^{*}\right)\right) \\
& =\frac{1}{2}\left(\left((\cdot)^{*} e_{b}\right) e_{a}+\left(e_{b}^{*} \cdot\right) e_{a}\right) .
\end{aligned}
$$


Due to the alternativity of the division algebras we also have

$$
e_{a}\left(e_{b}^{*} \cdot\right)+e_{a}\left((\cdot)^{*} e_{b}\right)=\left(e_{a} e_{b}^{*}\right)(\cdot)+\left(e_{a}(\cdot)^{*}\right) e_{b},
$$

and similarly for the other four possibilities above.

\section{Octonionic spinors in $D=11$}

In $D=11$ the Majorana spinor may be written as a 32 -component real column vector. However, if we consider $\mathbb{R}^{32}$ as the tensor product $\mathbb{R}^{4} \otimes \mathbb{R}^{8} \cong \mathbb{R}^{4} \otimes \mathbb{O}$ then we can write this as a 4-component octonionic column vector

$$
\lambda=\left(\begin{array}{c}
\lambda_{1} \\
\lambda_{2} \\
\lambda_{3} \\
\lambda_{4}
\end{array}\right), \quad \lambda_{\alpha} \in \mathbb{O}, \quad \alpha=1,2,3,4 .
$$

A natural set of generators $\left\{\gamma^{M}\right\}=\left\{\gamma^{0}, \gamma^{a+1}, \gamma^{9}, \gamma^{10}\right\}, M=0,1, \ldots, 10$ for the $4 \times 4$ octonionic Clifford algebra is then given by

$$
\begin{aligned}
& \gamma^{0}=\left(\begin{array}{cccc}
0 & 0 & 1 & 0 \\
0 & 0 & 0 & 1 \\
-1 & 0 & 0 & 0 \\
0 & -1 & 0 & 0
\end{array}\right), \quad \gamma^{a+1}=\left(\begin{array}{cccc}
0 & 0 & 0 & e_{a}^{*} \\
0 & 0 & e_{a} & 0 \\
0 & e_{a}^{*} & 0 & 0 \\
e_{a} & 0 & 0 & 0
\end{array}\right) \\
& \gamma^{9}=\left(\begin{array}{cccc}
0 & 0 & 1 & 0 \\
0 & 0 & 0 & -1 \\
1 & 0 & 0 & 0 \\
0 & -1 & 0 & 0
\end{array}\right), \quad \gamma^{10}=\left(\begin{array}{cccc}
1 & 0 & 0 & 0 \\
0 & 1 & 0 & 0 \\
0 & 0 & -1 & 0 \\
0 & 0 & 0 & -1
\end{array}\right) \text {, }
\end{aligned}
$$

with $a=0,1, \ldots, 7$. These matrices satisfy

$$
\gamma^{M} \gamma^{N}+\gamma^{N} \gamma^{M}=2 \eta^{M N} \mathbb{1}
$$

and the infinitesimal Lorentz transformation of the spinor $\lambda$ is

$$
\delta \lambda=\frac{1}{4} \omega_{M N} \gamma^{M}\left(\gamma^{N} \lambda\right),
$$

where $\omega_{M N}=-\omega_{N M}$. In general, the action of the rank $r$ Clifford algebra element on $\lambda$ can be written

$$
\gamma^{\left[M_{1}\right.}\left(\gamma^{M_{2}}\left(\ldots\left(\gamma^{M_{r-1}}\left(\gamma^{\left.M_{r}\right]} \lambda\right)\right) \ldots\right)\right)
$$

The positioning of the brackets in the above expression follows from repeated application of (2.7); non-associativity matters only for the imaginary gamma matrices $\gamma^{i+1}$, which provide a representation of the $\mathrm{SO}(7)$ Clifford algebra. If we define an operator $\hat{\gamma}^{M}$, whose action is left-multiplication by $\gamma^{M}$, then we can think of the rank $r$ Clifford algebra element as the operator

$$
\hat{\gamma}^{\left[M_{1} M_{2} \ldots M_{r}\right]} \equiv \hat{\gamma}^{\left[M_{1}\right.} \hat{\gamma}^{M_{2}} \ldots \hat{\gamma}^{\left.M_{r}\right]}
$$


where the operators $\hat{\gamma}^{M}$ must be composed as

$$
\hat{\gamma}^{M} \hat{\gamma}^{N} \lambda=\gamma^{M}\left(\gamma^{N} \lambda\right) \neq\left(\gamma^{M} \gamma^{N}\right) \lambda
$$

This ensures that the action of $\hat{\gamma}^{\left[M_{1} M_{2} \ldots M_{r}\right]}$ on a spinor is given by (4.5), as required.

\section{The octonionic M-algebra}

The anti-commutator of two supercharges in the $D=11$ supergravity theory is conventionally written as the 'M-algebra' $[18,19]$

$$
\left\{Q_{\bar{\alpha}}, Q_{\bar{\beta}}\right\}=\left(\gamma^{M} C\right)_{\bar{\alpha} \bar{\beta}} P_{M}+\left(\gamma^{M N} C\right)_{\bar{\alpha} \bar{\beta}} Z_{M N}+\left(\gamma^{M N P Q R} C\right)_{\bar{\alpha} \bar{\beta}} Z_{M N P Q R},
$$

where $\bar{\alpha}, \bar{\beta}=1, \ldots, 32, P_{M}$ is the generator of translations and $Z_{M N}$ and $Z_{M N P Q R}$ are the brane charges. The charge conjugation matrix $C_{\bar{\alpha} \bar{\beta}}$ serves to lower an index on each of the gamma matrices.

The left-hand side is a symmetric $32 \times 32$ matrix with 528 components, while the terms on the right-hand side consist of the rank 1,2 and 5 Clifford algebra elements, which form a basis for such symmetric matrices. In terms of $\mathrm{SO}(1,10)$ representations:

$$
(32 \times 32)_{\text {Sym }}=11+55+462 .
$$

We would like to write this algebra in terms of $4 \times 4$ octonionic matrices. However, the space of octonionic $4 \times 4$ matrices is of dimension $16 \times 8=128$, and hence naively does not carry nearly enough degrees of freedom to write (5.1).

The solution to this problem is to use the octonionic Clifford algebra operators $\hat{\gamma}^{\left[M_{1} M_{2} \ldots M_{r}\right]}$ defined in the previous section. These operators (including all ranks $r$ ) span a space of dimension $32 \times 32=1024$. In other words, their octonionic matrix elements are

$$
\left\langle e_{a} \mid \hat{\gamma}_{\alpha}^{M}{ }_{\alpha} e_{b}\right\rangle=\gamma_{\alpha a}^{M b}, \quad \alpha, \beta=1,2,3,4,
$$

and if we think of $\alpha a$ as a composite spinor index $\bar{\alpha}=1, \ldots, 32$, then the set of $\left\{\gamma^{M} \bar{\alpha}^{\bar{\beta}}\right\}$ generates the usual real Clifford algebra as in (5.1).

For the charge conjugation matrix, we define the $4 \times 4$ real matrix (which is numerically equal to $\gamma^{0}$ but with a different index structure)

$$
C_{\alpha \beta}=\left(\begin{array}{cccc}
0 & 0 & 1 & 0 \\
0 & 0 & 0 & 1 \\
-1 & 0 & 0 & 0 \\
0 & -1 & 0 & 0
\end{array}\right)
$$

The octonionic matrix elements of this are then trivially

$$
C_{\alpha a \beta b}=\left\langle e_{a} \mid C_{\alpha \beta} e_{b}\right\rangle=C_{\alpha \beta} \delta_{a b},
$$

which can be identified with the $32 \times 32$ matrix:

$$
C_{\bar{\alpha} \bar{\beta}}=C_{\alpha a \beta b}=C_{\alpha \beta} \delta_{a b} .
$$


Armed with these tools, the right-hand side can then be written over $\mathbb{O}$ simply by replacing $\bar{\alpha} \rightarrow \alpha$ and putting hats on the gammas:

$$
\left(\hat{\gamma}^{M} C\right)_{\alpha \beta} P_{M}+\left(\hat{\gamma}^{M N} C\right)_{\alpha \beta} Z_{M N}+\left(\hat{\gamma}^{M N P Q R} C\right)_{\alpha \beta} Z_{M N P Q R}
$$

With the identification $\bar{\alpha}=\alpha a$ we can also write the left-hand side of (5.1) in terms of the composite indices:

$$
\left\{Q_{\bar{\alpha}}, Q_{\bar{\beta}}\right\}=\left\{Q_{\alpha a}, Q_{\beta b}\right\} .
$$

Now, the expression (5.7) is an octonionic operator with matrix elements as on the righthand side of (5.1), so on the left we require an octonionic operator

$$
\left\{\widehat{Q_{\alpha}, Q_{\beta}}\right\}
$$

with matrix elements given by (5.8). The required operator is obtained simply by contracting (5.8) with the outer product $e_{a} \times e_{b}$ defined in (3.5):

$$
\left\{\widehat{Q_{\alpha}, Q_{\beta}}\right\} \equiv\left\{Q_{\alpha a}, Q_{\beta b}\right\} e_{a} \times e_{b}
$$

The octonionic formulation of the M-algebra is then

$$
\left\{\widehat{Q_{\alpha}, Q_{\beta}}\right\}=\left(\hat{\gamma}^{M} C\right)_{\alpha \beta} P_{M}+\left(\hat{\gamma}^{M N} C\right)_{\alpha \beta} Z_{M N}+\left(\hat{\gamma}^{M N P Q R} C\right)_{\alpha \beta} Z_{M N P Q R} .
$$

Using the first two versions of the outer product given in (3.6), we could write the left-hand side as

$$
\left\{\widehat{Q_{\alpha}, Q_{\beta}}\right\}=\frac{1}{2}\left(\left(Q_{\alpha} Q_{\beta}^{*}\right)(\cdot)+(\cdot)\left(Q_{\beta}^{*} Q_{\alpha}\right)+\left(Q_{\alpha}(\cdot)^{*}\right) Q_{\beta}+Q_{\beta}\left((\cdot)^{*} Q_{\alpha}\right)\right) .
$$

The first two terms look similar to the more intuitive anti-commutator $\left\{Q_{\alpha}, Q_{\beta}^{*}\right\}$, explored in [15], but to reproduce the full M-algebra we require all four terms above.

\section{Relation to lower dimensions}

It is interesting to consider the octonionic version of the supersymmetry algebra after an $\mathbf{1 1}=\mathbf{4}+\mathbf{7}$ split:

$$
\mathrm{SO}(1,10) \supset \mathrm{SO}(1,3) \times \mathrm{SO}(7) .
$$

Seven of the Clifford algebra generators $\gamma^{i+1}$ are imaginary, while the other four are real. This suggests that we split the dimensions as follows:

$$
\begin{aligned}
M=0,1, \ldots, 10 \rightarrow i+1 & =1, \ldots, 8 \\
\mu & =0,1,9,10 .
\end{aligned}
$$

In $D=4$ we regard the $D=11$ octonionic spinor $Q_{\alpha a} e_{a}$ as eight 4-component Majorana spinors $Q_{\alpha a}$, which we may leave packaged as an 'internal' octonion. This transforms as the spinor 8 of $\mathrm{SO}(7)$. The $D=4$ interpretation of the octonionic gamma matrices is as follows:

$$
\hat{\gamma}_{i+1}=\gamma_{*} \hat{e}_{i}
$$




\begin{tabular}{|c|cccc|}
\hline$D \backslash \mathcal{N}$ & 1 & 2 & 4 & 8 \\
\hline 11 & $\mathbb{O}^{4}$ & & & \\
7 & $\mathbb{H}^{4}$ & $\mathbb{O}^{4}$ & & \\
5 & $\mathbb{C}^{4}$ & $\mathbb{H}^{4}$ & $\mathbb{O}^{4}$ & \\
4 & $\mathbb{R}^{4}$ & $\mathbb{C}^{4}$ & $\mathbb{H}^{4}$ & $\mathbb{O}^{4}$ \\
\hline
\end{tabular}

Table 1. A summary of the division algebraic parameterisation of spinors used in $D=n+3$ supersymmetry algebras. Note that supersymmetry algebras sharing the same $\mathbb{A}$ are equivalent and that Cayley-Dickson doubling $\mathbb{A}$ corresponds to doubling $\mathcal{N}$, or equivalently climbing upwards in dimension $D$.

where $\hat{e}_{i}$ denotes the operator whose action is left-multiplication by $e_{i}$ and $\gamma_{*}$ (otherwise known as $\gamma_{5}$ ) is the highest rank Clifford element:

$$
\gamma_{*}=-\gamma^{0} \gamma^{1} \gamma^{9} \gamma^{10}=\left(\begin{array}{cccc}
0 & 0 & 0 & -1 \\
0 & 0 & 1 & 0 \\
0 & -1 & 0 & 0 \\
1 & 0 & 0 & 0
\end{array}\right)
$$

The matrix $C_{\alpha \beta}$ is just the charge conjugation matrix in $D=4$.

We do not split the $M, N$ indices of equation (5.11) into $\mu$ and $i$ parts here, as the expression of the right-hand side itself is not particularly illuminating. The result is a copy of the $\mathcal{N}=8$ supersymmetry algebra written over the octonions. The interesting point is that the $D=11$ supersymmetry algebra can be reinterpreted as an octonionic $D=4$ algebra.

More generally, the spinor and associated gamma matrices defined in (4.1) and (4.2) correspond to those of $D=4,5,7$ if we replace $\mathbb{O}$ with $\mathbb{R}, \mathbb{C}, \mathbb{H}$, respectively - see table 1 . This means that in this framework the minimal supersymmetry algebra in these dimensions is written over $\mathbb{R}, \mathbb{C}, \mathbb{H}$, while doubling the amount of supersymmetry corresponds to Cayley-Dickson doubling the division algebra. This process terminates when we reach maximal supersymmetry, i.e. when the Cayley-Dickson process takes us to $\mathbb{O}$, the largest normed division algebra.

The above discussion serves to emphasise the correspondence between the octonions and maximal supersymmetry in various dimensions. Rather than thinking of the M-theory algebra as an eleven-dimensional real algebra, it may be fruitful to think of it as a fourdimensional octonionic one, as in table 2 .

\section{Acknowledgments}

The work of LB is supported by an Imperial College Junior Research Fellowship. The work of MJD is supported by the STFC under rolling grant ST/G000743/1. 


\begin{tabular}{|c|cccc|}
\hline$D \backslash \mathcal{N}$ & 1 & 2 & 4 & 8 \\
\hline 11 & $\mathbb{R}^{32}$ & & & \\
7 & $\mathbb{R}^{16}$ & $\mathbb{C}^{16}$ & & \\
5 & $\mathbb{R}^{8}$ & $\mathbb{C}^{8}$ & $\mathbb{H}^{8}$ & \\
4 & $\mathbb{R}^{4}$ & $\mathbb{C}^{4}$ & $\mathbb{H}^{4}$ & $\mathbb{O}^{4}$ \\
\hline
\end{tabular}

Table 2. An alternative parameterisation of spinors used in $D=n+3$ supersymmetry algebras. From this point of view the octonions single out $D=4$.

Open Access. This article is distributed under the terms of the Creative Commons Attribution License (CC-BY 4.0), which permits any use, distribution and reproduction in any medium, provided the original author(s) and source are credited.

\section{References}

[1] M. Günaydin, Exceptional Realizations of Lorentz Group: Supersymmetries and Leptons, Nuovo Cim. A 29 (1975) 467 [inSPIRE].

[2] T. Kugo and P.K. Townsend, Supersymmetry and the Division Algebras, Nucl. Phys. B 221 (1983) 357 [INSPIRE].

[3] M.J. Duff, Supermembranes: The First Fifteen Weeks, Class. Quant. Grav. 5 (1988) 189 [INSPIRE].

[4] C.A. Manogue and J. Schray, Finite Lorentz transformations, automorphisms and division algebras, J. Math. Phys. 34 (1993) 3746 [hep-th/9302044] [INSPIRE].

[5] J.C. Baez and J. Huerta, Division Algebras and Supersymmetry I, arXiv:0909.0551 [INSPIRE].

[6] J.C. Baez and J. Huerta, Division Algebras and Supersymmetry II, Adv. Theor. Math. Phys. 15 (2011) 1373 [arXiv:1003.3436] [INSPIRE].

[7] J.M. Evans, Auxiliary fields for super Yang-Mills from division algebras, Lect. Notes Phys. 447 (1995) 218 [hep-th/9410239] [INSPIRE].

[8] L. Borsten, D. Dahanayake, M.J. Duff, H. Ebrahim and W. Rubens, Black Holes, Qubits and Octonions, Phys. Rept. 471 (2009) 113 [arXiv:0809.4685] [INSPIRE].

[9] A. Anastasiou, L. Borsten, M.J. Duff, L.J. Hughes and S. Nagy, Super Yang-Mills, division algebras and triality, JHEP 08 (2014) 080 [arXiv:1309.0546] [INSPIRE].

[10] L. Borsten, M.J. Duff, L.J. Hughes and S. Nagy, A magic square from Yang-Mills squared, Phys. Rev. Lett. 112 (2014) 131601 [arXiv:1301.4176] [INSPIRE].

[11] A. Anastasiou, L. Borsten, M.J. Duff, L.J. Hughes and S. Nagy, A magic pyramid of supergravities, JHEP 04 (2014) 178 [arXiv:1312.6523] [INSPIRE].

[12] E. Cremmer and B. Julia, The $N=8$ Supergravity Theory. 1. The Lagrangian, Phys. Lett. B 80 (1978) 48 [INSPIRE].

[13] M.J. Duff and J.X. Lu, Duality Rotations in Membrane Theory, Nucl. Phys. B 347 (1990) 394 [INSPIRE]. 
[14] C.M. Hull and P.K. Townsend, Unity of superstring dualities, Nucl. Phys. B 438 (1995) 109 [hep-th/9410167] [INSPIRE].

[15] F. Toppan, On the Octonionic M-algebra and superconformal M-algebra, Int. J. Mod. Phys. A 18 (2003) 2135 [hep-th/0307070] [INSPIRE].

[16] J.C. Baez, The Octonions, Bull. Am. Math. Soc. 39 (2002) 145 [math/0105155] [InSPIRE].

[17] S. De Leo and K. Abdel-Khalek, Octonionic representations of $G L(8, R)$ and $G L(4, C), J$. Math. Phys. 38 (1997) 582 [hep-th/9607140] [InSPIRE].

[18] J.W. van Holten and A. Van Proeyen, $N=1$ Supersymmetry Algebras in $D=2, D=3$, $D=4 \bmod 8, J$. Phys. A 15 (1982) 3763 [INSPIRE].

[19] P.K. Townsend, P-brane democracy, hep-th/9507048 [INSPIRE]. 